\title{
Chemical characterization of wound ointment (WO) and its effects on fracture repair: a rabbit model
}

\author{
Zhixue Ou ${ }^{1 \dagger}$, Qi Cheng ${ }^{2 \dagger}$, Yueping Chen ${ }^{1}$, Tao Chen ${ }^{1}$, Xiangbin Rong ${ }^{1}$, Feipan Long ${ }^{1}$, Xiaoyun Zhang ${ }^{1}$, \\ Qinghua Liang ${ }^{1}$ and Zhe Feng ${ }^{1 *}$
}

\begin{abstract}
Background: Wound ointment (WO), a kind of Chinese medicine, can significantly promote fracture healing. The study aimed at analyzing the chemical composition and the effects of WO on fracture of rabbits and tried to explore the corresponding molecular mechanism in cytokine.
\end{abstract}

Methods: The qualitative and quantitative analysis of WO was conducted by liquid chromatography-mass spectrometry (LC-MS). Fifty-four Zealand mature male rabbits were randomly divided into 3 groups: Control group, Yunnan Baiyao (YB) group and WO group. All the rabbits suffered a fracture of right radius and were then stabilized with an external fixator. Treated with different methods, fracture healing was observed. The bone specimens were subjected to radiograph, immunohistochemistry (IHC) analysis, hematoxylin-eosin staining ( $\mathrm{HE})$, western blot and enzyme linked immunosorbent assay (ELISA).

Results: A total of 12 active compositions were detected by LC-MS. Radiographs showed a considerably better bone healing and remodeling of the fracture in WO group. HE experiments showed that a large number of osteoclasts appeared in the early stage when treated with WO. In immunohistochemistry (IHC), western blot and ELISA test, significant increases in vascular endothelial growth factor (VEGF) expression were observed in WO group compared with other two groups.

Conclusions: Wound ointment contained active compositions which efficiently promoted fracture healing through increasing the expression of VEGF.

Trial Registration Not applicable

Keywords: Animals, Osteoclasts, Fracture healing, Wound ointment (WO), Radius fracture, VEGF

\section{Background}

Bone fracture is a very common disease, which is usually caused by high force, stress, or a minimal trauma injury. An average fracture incidence of $1.17 \%$ in males and $1.07 \%$ in females has been reported in UK and over 6 million cases occurred in the United States per year [14]. Fracture healing is a complicated process, including

\footnotetext{
*Correspondence: chizf0618@163.com

†Zhixue Ou and Qi Cheng contributed equally to this work

${ }^{1}$ Department of Traumatic Orthopedics and Hand Surgery, Ruikang Hospital Affiliated to Guangxi University of Chinese Medicine, Block B, No 10 Huadong Road, Nanning 530011, Guangxi, China

Full list of author information is available at the end of the article
}

granulation tissue formation, cartilage callus formation, lamellar bone deposition and remodeling to original bone contour. Surgical management is the most common treatment for fractures, while there is still a risk of morbidity and mortality among the elderly population [5]. Thus, finding a safer and more efficient treatment for fracture is essential. Vascular endothelial growth factor (VEGF) is a sub-family member of growth factors which are important to both vasculogenesis and angiogenesis process. In addition, it has been widely accepted that VEGF played a vital role in bone regeneration through accelerating the process of angiogenesis, which has been confirmed through several animal models as well $[6,7]$. 
There are sufficient evidences supporting that the high expression of VEGF protein can accelerate the healing of femoral fractures in mice and remedy radius segmental defects in rabbits [8, 9].

Yunnan Baiyao (YNBY) is one of the most popular prescriptions of Chinese herbal medicine which possesses haemostatic functions of both open and closed wounds. It is deemed as "a must for first aid" therapy in China of both internal and external hemorrhage as well as open and closed wounds due to its renowned haemostatic functions. It was also reported that YNBY can effectively reduce the severity of murine experimental colitis by both immune-suppressing and wound-healing mechanisms [10-12].

Wound ointment (WO) is made from Chinese medicinal herbs, containing panax, notoginseng, drynaria fortunei and rhubarb etc. Those herbal remedies have active compositions with synergism to relieve pain, prevent infection, and promote healing with less side effects. Antibacterial constituents and analgesic were added in several WOs to bitterly treat wounds and prevent infection. Some uncommon ointments enriching vitamins, including vitamin $E$, vitamin $A$ and vitamin $\mathrm{D}$ can promote skin healing from scrapes, cuts and burns.

The main usage of WOs is to treat soft tissue injury, accelerate blood circulation and relieve congestion, swelling and pain. WO also has anti-inflammatory and antibacterial effects with non-stimulation to skin, heart, liver, kidney and other organs. After traditional disinfection, WO may be dressed directly, or painted onto gauze. It was first applied for fracture regeneration by Chinese medicine doctors $[13,14]$. With a clinical application for 30 years, WO has its positive effects on the union of bone confirmed, and exerted a certain influence in China and Southeast Asia $[15,16]$. However, there was no relative animal experiment or clinical study to evaluate its efficacy on fracture healing systematically, and its functional mechanism remains unknown to the public due to a lack of researches. These factors mentioned above have largely limited the wide application of WO in clinic. Thus, in this research, we utilized rabbits for radius fracture model to investigate the effects of $\mathrm{WO}$ on fracture healing and illustrate the potential mechanisms of WO.

\section{Methods}

\section{Preparation of sample solutions}

A total of $4.0 \mathrm{~g}$ WO sample was extracted with $40 \mathrm{~mL}$ methanol using Soxhlet extractor for $12 \mathrm{~h}$ until the complete extraction. Afterwards, the solution was filtered and evaporated. The crude remnants were uniformly mixed with $5 \mathrm{~mL}$ methanol for further analysis.

\section{Analysis of liquid chromatography-mass spectrometry (LC-MS)}

LC-MS was performed using Waters Acquity UPLC system (Waters, Milford, MA, USA) coupled with TOF mass spectrometer (Bruker, Bremen, Germany). The results were acquired using Hystar software (Bruker). Acquity UPLC BEH C18 column $(2.1 \mathrm{~mm} \times 100 \mathrm{~mm}$, $1.7 \mu \mathrm{m})$ served as the stationary phase, $0.1 \%$ formic acid in pure water (A) and $0.1 \%$ formic acid in acetonitrile (B) with a flow rate of $0.4 \mathrm{~mL} / \mathrm{min}$ were utilized as the mobile phase. The conditions were as follows: 0-1 min, $5 \% \mathrm{~B}$; $1-4 \mathrm{~min}, 20 \% \mathrm{~B}$; 4-6 min, 25\% B; 6-10 min, 40\% B; 10-12 min, $60 \%$ B; $12-15$ min, $100 \%$ B; $15-16$ min, $40 \%$ B; $16-20 \mathrm{~min}, 5 \% \mathrm{~B}$. The column temperature was maintained at $40{ }^{\circ} \mathrm{C}$, and the injection volume of each sample was $2 \mu \mathrm{L}$.

The mass spectrometer was operated in the negative mode, the scanning range of $\mathrm{m} / z$ from 100 to 3000 . The mass conditions were as follows: gas (N2) flow rate was $8 \mathrm{~L} / \mathrm{min}$ and the temperature was $180{ }^{\circ} \mathrm{C}$; the capillary voltage was $4500 \mathrm{~V}$.

\section{Animal model and fracture surgery}

Fifty-Four New Zealand white male rabbits (provided by Animal Center of Guangxi University of Chinese Medicine, Guangxi, China), which were 10 months old with body weight ranging from 2.0 to $2.5 \mathrm{~kg}$, were involved in this study. All rabbits were kept singly and fed with a standard laboratory diet and tap water under climatecontrolled conditions $\left(25^{\circ} \mathrm{C}\right.$; $55 \%$ humidity; $12 \mathrm{~h}$ of light alternating with $12 \mathrm{~h}$ of darkness). A small patch of skin dermis was excised from the back and the right forelimb of each rabbit. After the injection of anesthetic $(30 \mathrm{mg} /$ $\mathrm{mL}$, sodium pentobarbital sodium solution, SigmaAldrich, MO, USA) into the vein of the rabbit ear, all the following procedures were performed in disinfection area with rabbits in supine position. A $1.5 \mathrm{~cm}$ longitudinal incision was made to expose the middle part of radius. The periosteum was cut and peeled off, and then the radial shaft was exposed. A fracture model was established by bone sheared with peripheral vessel, muscle and cartilage tissue preserved, keeping the ulna integrate. The research was approved by the ethical committee of Ruikang Hospital Affiliated to Guangxi University of Chinese Medicine. The Minimum Standards of Reporting Checklist contained details of the experimental design, statistics and resources used in this study (Additional file 1).

\section{Grouping and treatment}

The rabbits were categorized into three groups $(n=18$ each group). Rabbits received normal saline only were in control group, those treated with Yunnan Baiyao (YB, Yunnan Baiyao group Co., Ltd., Yunnan, China) were 
regarded as YB group, and WO group consisted of rabbits treated with WO (Ruikang Hospital Affiliated to Guangxi University of Chinese Medicine, Guangxi, China). Five milligram/kilogram of $\mathrm{YB}$ and $\mathrm{WO}$ were externally applied on the fracture three times a day with bandage being put on and dressings were changed every 3 days. The fracture parts of control group were bandaged without any drug applied.

\section{Preparation and treatment of specimens}

The rabbits were killed at different time point $(3,7$, $14,21,28,35$ days after operation) by being injected 10-15 mL air into the syringes. After the skin of the fracture was cut immediately, the surrounding muscles, tendons and other soft tissues were stripped. Hyperplastic soft tissue, fibrous and bones callus were halved. One part was fixed in 10\% neutral formalin solution and prepared for the following HE stain and IHC analysis; other part was wrapped by tin foil and stored at $-80{ }^{\circ} \mathrm{C}$ for western blot. The sacrifice of rabbits and the preservation of the specimens were completed within $10 \mathrm{~min}$ to prevent protein degradation and cell autolysis rupture.

\section{Radiography analysis}

The procedure of X-ray and micro-CT observation was similar to the previous studies [17, 18]. Briefly, fracture morphology was detected by digital X-ray equipment (Siemens, Chicago, USA). Three-dimensional (3D) images were rendered. A laboratory micro-CT scanner (Scanco Medical AG, Brüttisellen, Switzerland) and the VGStudio MAX software (Dürr, Bietigheim-Bissingen, Germany) were used for the micro-CT examination. The proportion about total void area of all area in transverse section image was calculated, and the fraction of mineralized callus was quantified.

\section{Alkaline phosphatase (ALP) assay}

At each time point, $4 \mathrm{~mL}$ ear margin venous blood stewed in the bio-tube for $30 \mathrm{~min}$ was centrifugalized at the speed of $2500 \mathrm{r} / \mathrm{min}$. Then, the upper layer of the serum was removed and ALP activity of the lower layer was detected with ALP assay kit (Sigma-Aldrich, St. Louis, MO, USA). The measurement was performed using Beckman LX 20 Analyzer (Beckman Coulter, Inc., CA, USA).

\section{Hematoxylin-eosin (HE) staining}

The bone specimens were fixed in $10 \%$ formaldehyde solution for $48 \mathrm{~h}$ and decalcified in $15 \%$ ethylene diamine tetraacetic acid (EDTA) for 3 weeks. Then, the decalcified samples were embedded in paraffin, cut into $4 \mathrm{~mm}$-thick sections and dehydrated with gradient ethanol. After that, the sections were stained with $\mathrm{HE}$ and assessed with the optical microscope.

\section{Immunohistochemistry}

The bone specimen sections, which were dewaxed and rehydrated with xylene solution and gradient ethanol, were then hot fixed with $0.01 \mathrm{M}$ Citrate repair solution (pH 6.0) for $2 \mathrm{~min}$ in hyperbaric condition. $100 \mu \mathrm{L}$ antiVEGF receptor 1 (rabbit, 1: 800, ABcam, US) was added and incubated overnight. Then the sections were incubated with horseradish enzyme labeled goat anti-rabbit IgG (Beijing Zhong Jin Jinqiao company, Biological Technology Co., Ltd., Beijing, China) for $40 \mathrm{~min}$. Afterwards, the sections were treated with 3,3'-diaminobenzidine (DAB) coloring agent for $20 \mathrm{~s}$, hematoxylin re-infection for $1 \mathrm{~min}$ and dehydrated. The figures were analyzed with the MIQAS medical image quantitative analysis system (Motic China Group Co., Ltd., Guangdong, China), 5 fields of vision were selected randomly with about 100 cells in each field. In each field, mean optical density, the positive reaction area and total area of cells were measured (cells area was $0.15 \mathrm{~mm}^{2}$ ). Subsequently, the average rate of positive area was calculated.

\section{Western blot}

The harvested bone specimens were cut into pieces, weighed and grinded into powder in liquid nitrogen with radio-immunoprecipitation assay (RIPA) buffer. The total proteins were extracted using protein extraction kit (Millipore, Billerica, MA, USA). Equal weight of proteins were loaded and separated by sodium dodecyl sulfate-polyacrylamide gel electrophoresis (SDS-PAGE), transferred to polyvinylidene fluoride (PVDF) membranes and then blocked with 5\% skim milk at room temperature for $1 \mathrm{~h}$. Primary antibody anti-rabbit F-box protein 32 (FBXO32) diluted with Tris buffered saline solution (TBST, 1: 2500) was added to the membranes and incubated at $4{ }^{\circ} \mathrm{C}$ overnight. The membranes were then incubated with goat monoclonal anti-rabbit, the secondary antibody marked with horseradish peroxidase (HRP, 1: 2000), for $2 \mathrm{~h}$ at room temperature. Glyceraldehyde-3-phosphate dehydrogenase (GAPDH) served as the internal control. Signal detection was performed using Chemiluminescence reagent kit (Amersham, IL, USA). The antibodies mentioned above were all bought from Beijing Zhong Shan Jinqiao Biological Technology Co., Ltd. (Beijing, China).

\section{Enzyme linked immunosorbent assay (ELISA)}

The bone specimens were cut off and rinsed with precooled phosphate buffered solution (PBS) $(0.01 \mathrm{M}$, $\mathrm{pH}=7.4)$ to remove residual blood and weighted. The specific volume of PBS $(1: 9 \mathrm{w} / \mathrm{w})$ was added to a glass homogenizer and sufficiently grinded on ice. After $5000 \mathrm{~g}$ 
homogenate were ultrasonically broken and thawed, it was centrifuged for $10 \mathrm{~min}$ and the supernatant was collected. Rabbit VEGF specific enzyme-linked immunoassay kit (TWp027731, TongWei, Shanghai, China) was used to detect the VEGF expression level. Manufacturer's protocol was strictly followed and the optical density (OD) was measured at $450 \mathrm{~nm}$.

\section{Statistical analysis}

All statistical analyses were performed by SPSS 19.0 software (SPSS, IL, USA). The significant differences in numerical data (mean $\pm \mathrm{SD}$ ) were estimated with the analysis of variance (ANOVA). Differences between two groups were analyzed by unpaired $t$ tests. $P$ value less than 0.05 was defined as statistical significance.

\section{Results}

\section{2 compositions were detected in WO}

As shown in Table 1, a total of 12 compositions were detected in WO. The structures of the active compounds were shown in Additional file 2: Figure S1. Most of compositions have the property of antioxidant, anti-inflammatory and antibacterial. Some compositions have been verified to play a crucial role in the fracture healing, especially asperosaponin VI, which could accelerate the proliferation of the bone marrow mesenchymal stem cells and their differentiation toward osteoblasts [19].

\section{Fracture healing was the fastest in WO group}

The control group showed the common process of fracture healing (Fig. 1), where the fracture line was still clearly observed 21 days after the operation. At the 35th day, the fracture line became blurry, and the bone callus formed. While in YB group, the speed of fracture healing was much faster than that of control group. At the

Table 1 Comprehensive analysis of WO by LC-MS

\begin{tabular}{lllll}
\hline $\begin{array}{l}\text { Retention time } \\
\text { (min) }\end{array}$ & $\boldsymbol{m} / \boldsymbol{z}$ & Mol. wt & Formula & Compounds \\
\hline 2.23 & 137 & 154.12 & $\mathrm{C}_{7} \mathrm{H}_{5} \mathrm{O}_{3}$ & Protocatechuic acid \\
2.81 & 289 & 308.28 & $\mathrm{C}_{15} \mathrm{H}_{14} \mathrm{O}_{6}$ & Catechin \\
2.86 & 137 & 138.12 & $\mathrm{C}_{7} \mathrm{H}_{6} \mathrm{O}_{3}$ & $\begin{array}{l}\text { p-Hydroxybenzoic } \\
\text { acid }\end{array}$ \\
4.55 & 595 & 596.53 & $\mathrm{C}_{27} \mathrm{H}_{32} \mathrm{O}_{15}$ & Neoeriocitrin \\
5.27 & 579 & 580.53 & $\mathrm{C}_{27} \mathrm{H}_{32} \mathrm{O}_{14}$ & Naringin \\
7.98 & 269 & 270.23 & $\mathrm{C}_{15} \mathrm{H}_{10} \mathrm{O}_{5}$ & Emodin \\
9.36 & 928 & 929.1 & $\mathrm{C}_{47} \mathrm{H}_{76} \mathrm{O}_{18}$ & Asperosaponin Vl \\
10.02 & 266 & 283.21 & $\mathrm{C}_{15} \mathrm{H}_{7} \mathrm{O}_{6}$ & Rhein \\
10.32 & 271 & 272.25 & $\mathrm{C}_{15} \mathrm{H}_{12} \mathrm{O}_{5}$ & Naringenin \\
11.4 & 285 & 286.23 & $\mathrm{C}_{15} \mathrm{H}_{10} \mathrm{O}_{6}$ & Kaempferol \\
12.63 & 283 & 284.27 & $\mathrm{C}_{16} \mathrm{H}_{12} \mathrm{O}_{5}$ & Physcion \\
13.29 & 861 & 862.75 & $\mathrm{C}_{42} \mathrm{H}_{38} \mathrm{O}_{20}$ & Sennoside A \\
\hline
\end{tabular}

21st day, the fracture line was fuzzy with massive callus appeared at the fracture end. At the last observation in day 35 , the fracture line was almost disappeared, but fracture ghosting still existed. In WO group, the recovery rate of fractured bone was faster than that of other two groups. At the 21st day, the obscure space was very narrow with massive bone callus proliferated. At the 35th day, the fracture line disappeared and the fractured limbed was almost repaired. As shown in Fig. 2, micro-CT results further verified the healing progress and presented more distinct fracture healing condition. The YB group and WO group were much superior to that of control group. In the WO group, the fracture was almost recovered at the 35th day. As bone volume shown in Table 2, the volume of YB and WO group had significant difference compared with that of control group. WO group also showed larger volume in YB group in the 21st day. The results showed that $\mathrm{WO}$ and $\mathrm{YB}$ could promote the fracture healing in a short time, and the WO had the better effect during the interim period.

\section{Tissues grew fastest in WO group}

As shown in Fig. 3, at the 7th day, tissue necrosis and reactive periosteum hyperplasia occurred in control group, with inflammatory cell gathered and capillary proliferated. At the 14th day, little fibrocyte formed with slight reaction while at the 21st day, the proliferation became active and at the 35th day, the bone callus joint with no woven bone molded. As in YB group, there was periosteal reaction and proliferation, and fibroblast gathered obviously at the 7 th day. The chondrocyte appeared the 14th day and proliferated at the 21st day with primary callus formed, when subperiosteum ossification, endochondral ossification and primitive trabecula bone could be observed at the fracture end. At the 28th day, fracture was connected with a great deal of woven bones formed. At the last observation, primary marrow cavity appeared, but there was no connection between the upper and lower cavum ossis. Meanwhile, the bone recovery process in $\mathrm{WO}$ group was similar to that in $\mathrm{YB}$ group during the first 14 days. However, massive bone callus appeared and connected at the 21st day, primary marrow cavity formed at the 28th day and both ends of bone medullary cavity were connected in the last examination. In summary, the WO group showed the best bone healing effect.

\section{ALP concentration was the highest in WO group}

ALP activity is an indicator that measures the selfrepaired ability. Osteon recovery is strongly associated with the increase of ALP. As shown in Fig. 4, the value of ALP in serum showed an upward tendency in three groups. ALP value in WO group increased significantly compared with the control group $(P<0.01)$ at the 28th 


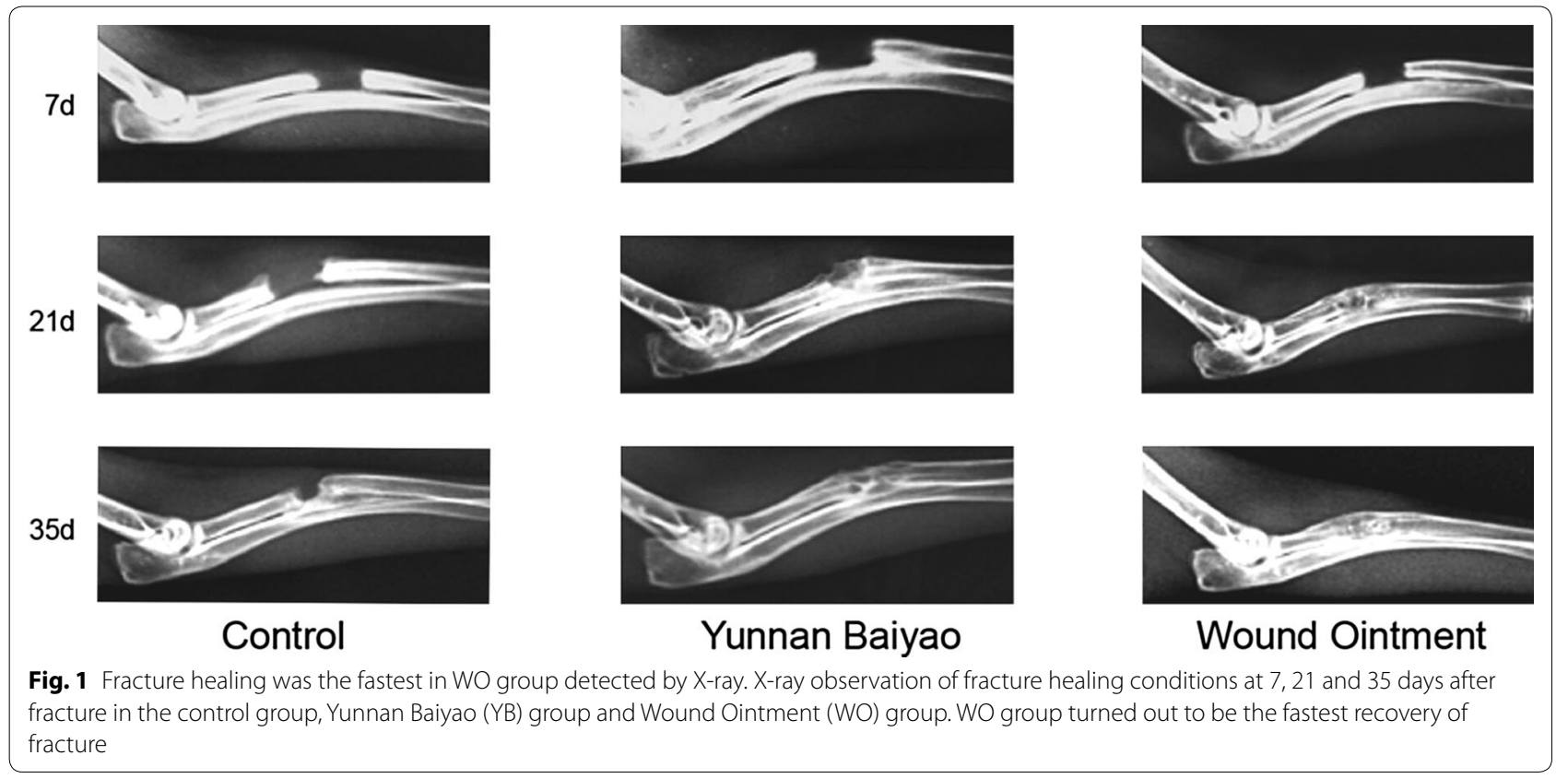

day, the relevant phenomena was the surprising speed of bone formation. 35 day after the operation, the ALP level in the YB group and WO group both showed the significant increase compared with the control group $(P<0.01)$.

\section{WO accelerated fracture healing by promoting the release of VEGF}

The positive substance was located in cytoplasm, in other words, the claybank cytoplasm showed a positive signal (Fig. 5). At the 7th day, in NC group, VEGF expressed high in dense fibrous connective tissue and expressed low in few hyperplastic chondrocytes. The expression of VEGF in YB was stronger than that in control group, and more chondrocytes showed positive VEGF signal. WO group showed the higher expression of VEGF in both the dense connective tissue and the chondrocytes compared with other two groups. In the 14th day, the proliferation of cartilage was active and VEGF was significantly increased while the hypertrophic chondrocytes were less and the contained VEGF was negative. In YB group, the new bone was arranged into trabeculae which was consisted of cartilage and fibrous bones and covered with osteoblasts. These osteoblasts showed positive expression of VEGF signal. Compared with YB group, WO group showed the similar results, while the number of osteoblasts was more and the positive VEGF signal was also stronger. At the 21st day, the typical trabeculae was found and covered a large number of osteoblasts containing VEGF. The number of osteoblasts significantly decreased, however, the size of callus increased in YB and WO group and there was no significant difference between them. After 28 days, there were more callus in control group showed negative expression of VEGF and the number of osteoblasts decreased. In YB and WO group, primary bone marrow cavity appeared and showed slightly positive expression of VGEF, and the expression level was higher in WO than in YB group. At the 35th day, the NC group was in the primary bone marrow cavity stage, $\mathrm{YB}$ group and WO group has developed more secondary bone marrow cavity which was relatively more in the WO group and showed higher positive expression of VEGF. The expressions of VEGF in YB group and WO group were much higher than that in control group at each time point $(P<0.01)$, and both reached the peak at the 28th days while the control group increased gradually during the monitoring time. In conclusion, we might speculate that WO promoted the release of VEGF in a short time.

\section{VEGF was highly expressed in WO group}

Results of western blot further confirmed the regular expression of VEGF protein in the fracture of rabbits during fracture healing in control group, YB group and

Table 2 The bone volume of UCT in 7, 21 and 35 days

\begin{tabular}{llll}
\hline & Control & YB & WO \\
\hline 7 days & $0.479 \pm 0.026$ & $0.627 \pm 0.047^{* *}$ & $0.855 \pm 0.057^{* * *}$ \\
21 days & $0.684 \pm 0.043$ & $0.942 \pm 0.061^{* * *}$ & $1.197 \pm 0.066^{* * * \# \#}$ \\
35 days & $0.875 \pm 0.056$ & $1.225 \pm 0.073^{* * *}$ & $1.301 \pm 0.105^{* * *}$ \\
\hline
\end{tabular}

** $P<0.01$, *** $P<0.001$. There was significant difference between the control group and two treatment group; ${ }^{\# \#} P<0.001$. There was significant difference between the YB group and WO group 


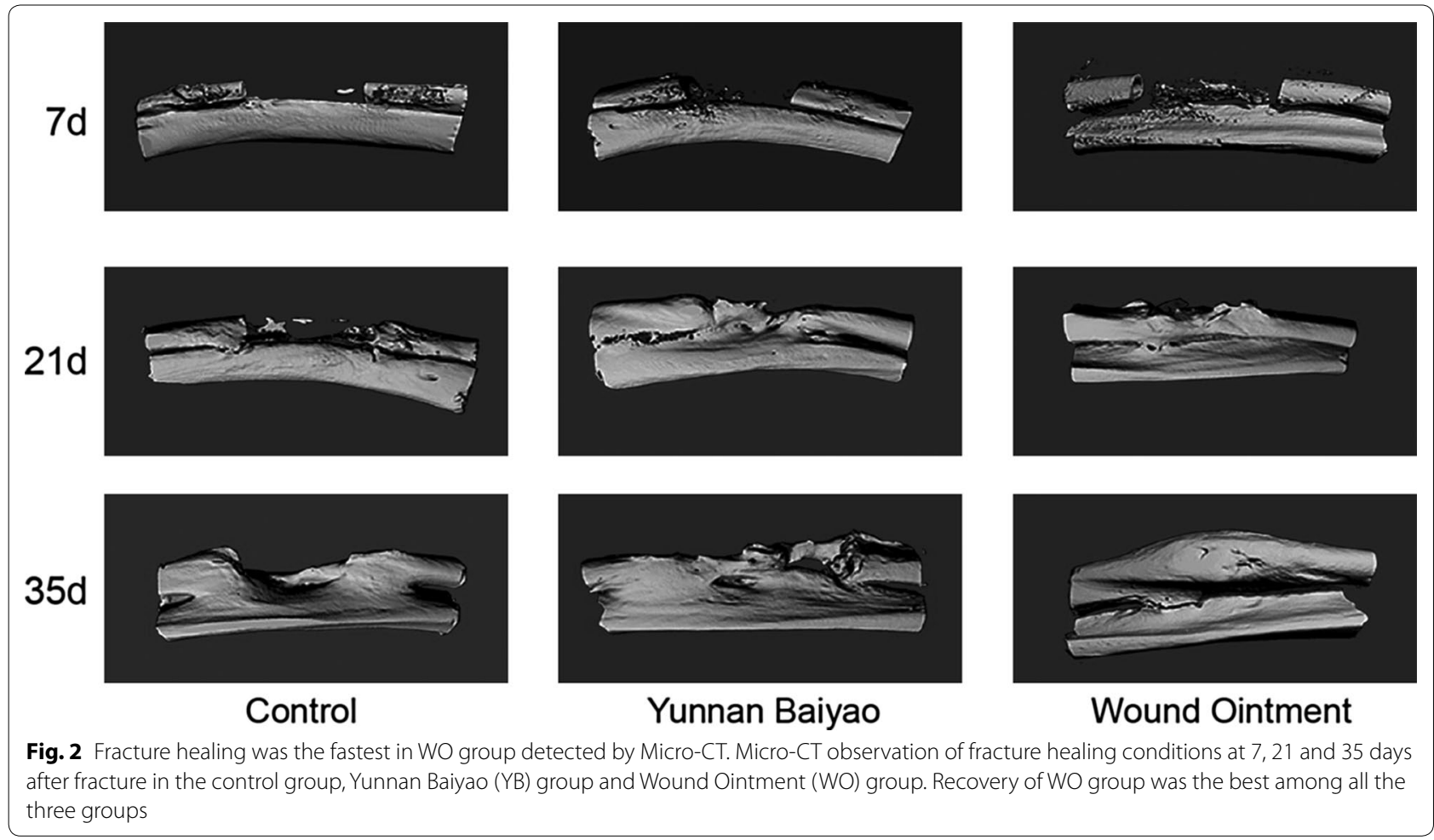

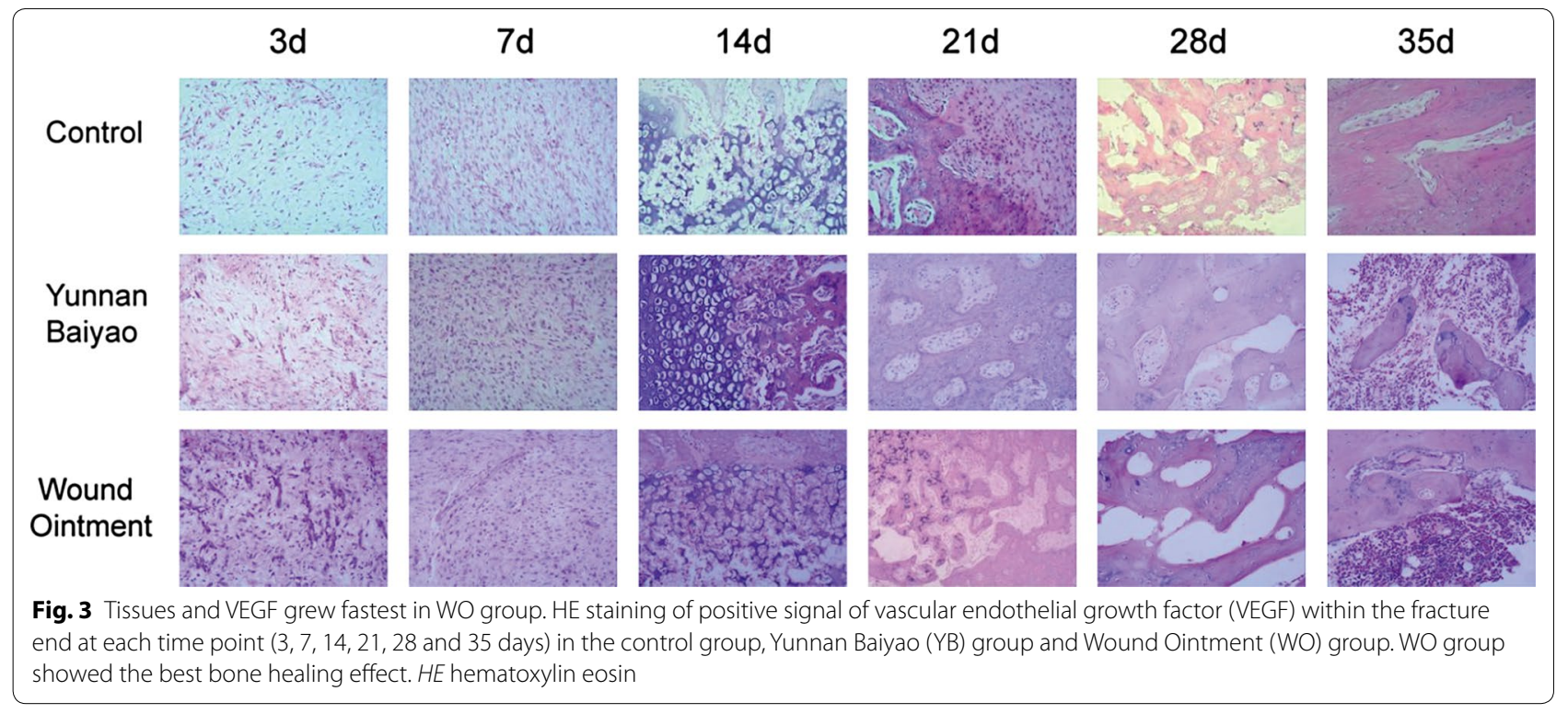

WO group (Fig. 6a). In the process of fracture healing, the expression level of VEGF in control group was the lowest but increased gradually. After 14 days, the growth was accelerated distinctly. In both YB group and WO group, the expressions of VEGF were augmented dramatically and peaked at the 28th day, and then dropped slightly. Overall, the VEGF expressions in YB and WO group were significantly higher than that in control group $(P<0.01)$. The results were consistent with the IHC analysis.

Results of ELISA confirmed that secretion of VEGF protein in control group, YB group and WO group 


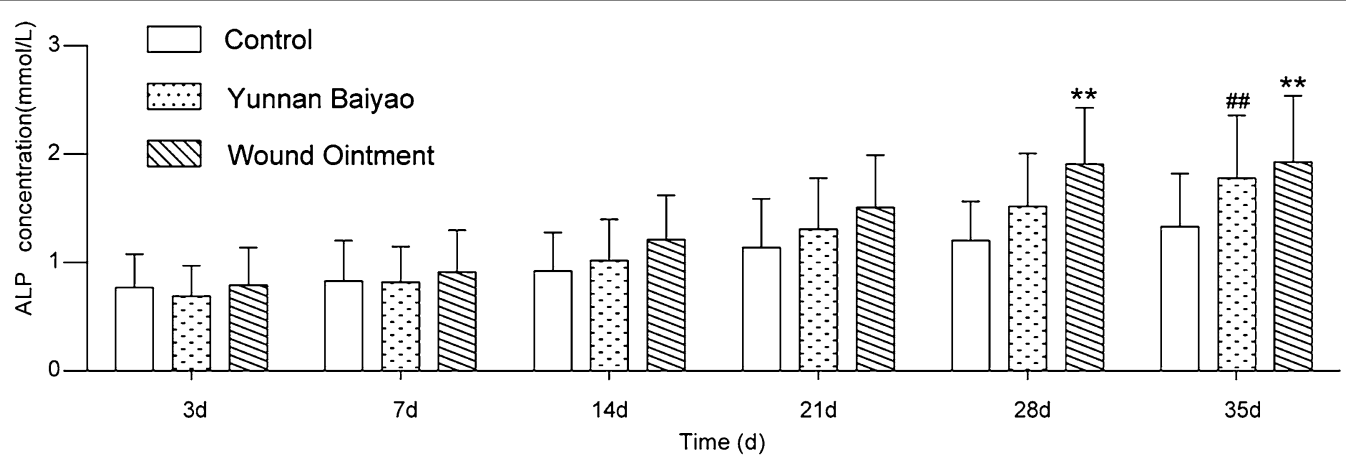

Fig. 4 ALP concentration was the highest in WO group. ALP assay detected the value of ALP in fracture tissue at each time point $(3,7,14,21,28$ and 35 days) in the control group, Yunnan Baiyao (YB) group and Wound Ointment (WO) group. WO group increased the fastest, suggesting a surprising speed of bone formation. ${ }^{\#} P<0.01, Y B$ group versus control group; ${ }^{*} P<0.01$, WO group versus control group. ALP alkaline phosphatase

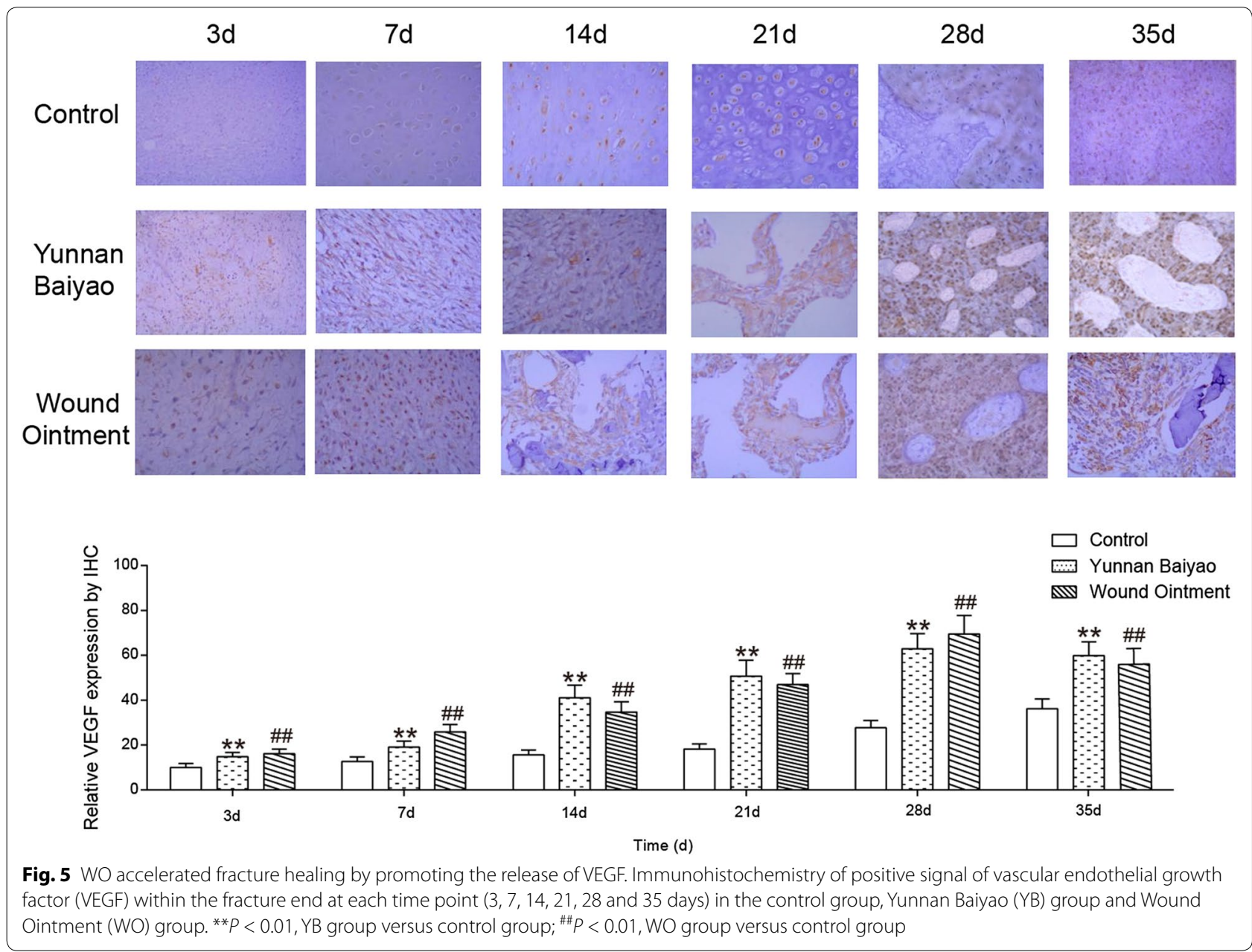

(Fig. 6b). The trend in the three groups in 35 days was coordinated with that of western blot. The expression of VEGF was higher in the WO group especially in the 28th day, nearly $3000 \mathrm{pg} / \mathrm{mL}$.

\section{Discussion}

A series of physiological processes are involved in bone fracture healing, each of which has fundamental roles in contributing to the healing process [20]. As one of the 


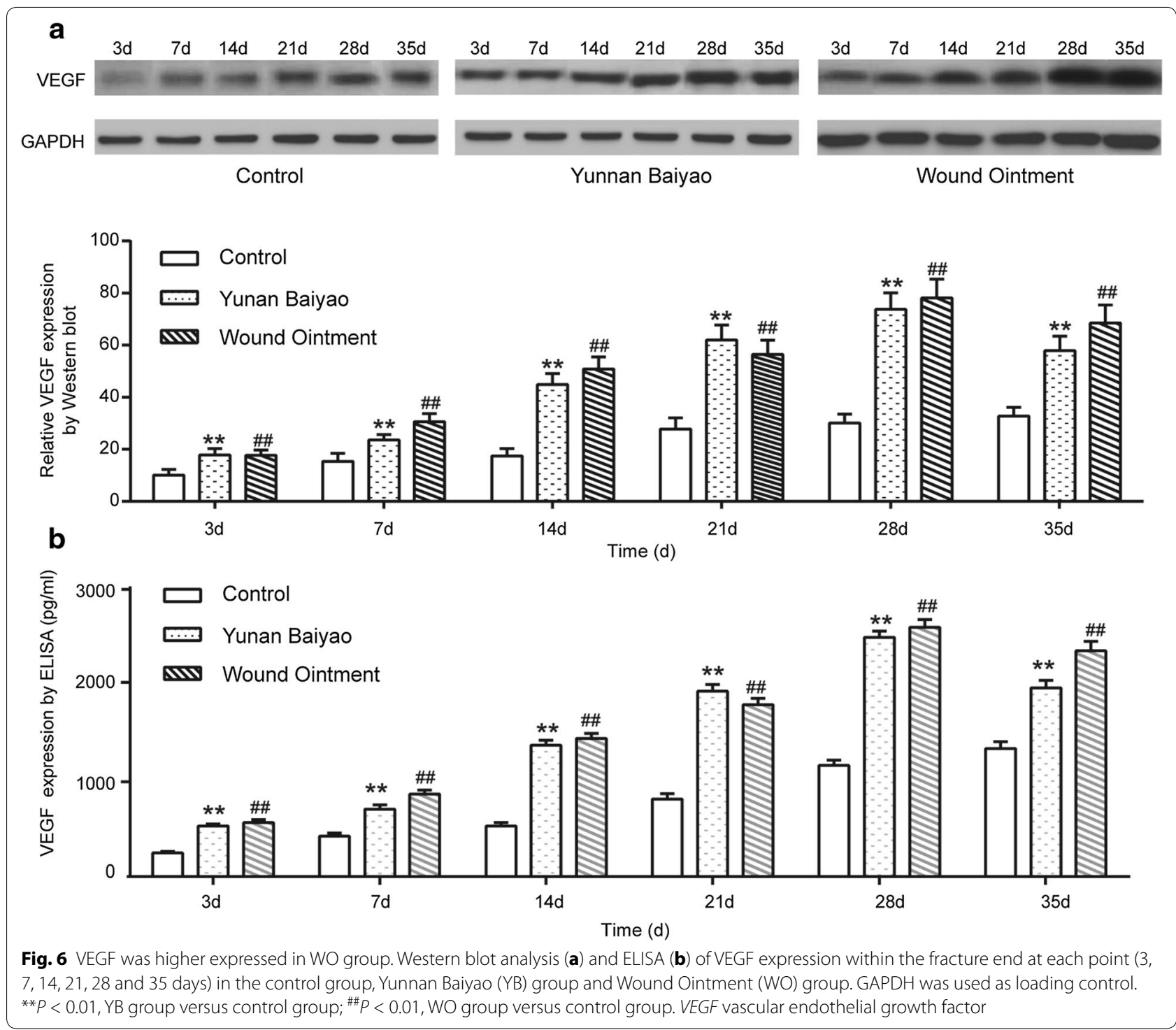

most important factors in influencing fracture healing, VEGF can stimulate local vascular regeneration in the fracture [21]. There are many studies showing that the application of VEGF can work effectively on angiogenesis and speed the process of bone repair [22-24]. Meanwhile, establishment of animal fracture model will be of great value in the understanding of fracture repair process [25]. The purpose of this research is to investigate whether WO could facilitate the expression of VEGF and accelerate fracture union. Our research was mainly based on a rabbit model of radius fracture. After treatment of Yunnan Baiyao or WO, the healing situation was assessed and compared with the control group. The experimental data showed that WO behaved well in promote neovascularization and callus regeneration via up-regulating the expression of VEGF, which contributed to accelerating fracture healing.
X-ray and micro-CT were logically applied to evaluate the fracture degree, bone strength, as well as fracture healing degree, because of their functions in quantitatively analyzing the microarchitecture of bones $[26,27]$. Our observations of X-ray and micro-CT in different time points revealed that in terms of the degree of callus formation and mineralization in fracture healing, WO group came out to be the best-performing one, followed by the YB group, and the control group was the worst.

Results of histomorphometric analysis in the three groups (control, YB and WO group) showed a similar bone formation process. The first change observed by light microscope was the regeneration of chondroblasts and the formation of hyaline cartilage. Loose connective tissue reverted to dense connective tissue gradually and developed into fibrous callus. With the proliferation, 
differentiation and growth of osteoblasts, fracture callus gradually replaced fibrous callus. At the last stage, osteoclasts became active. Fracture healing was in the phase of remodeling original bone contour. In WO group, the granulation tissue formation, chondroblasts and osteoblasts proliferation, fibrous callus and fracture callus remodeling process occurred at 3 or 7 days after fracture, which was faster than other groups. Significant cartilaginous osteogenesis and faster new bone growth were seen in WO group. These evidences indicated that WO could accelerate new bone growth through promoting cartilaginous osteogenesis. IHC analysis, Western blot and ELISA assay demonstrated that $\mathrm{YB}$ and WO played the same role in influencing VEGF expression, while VEGF showed a stronger expression in WO group than in YB group. When compared with control group, both WO and YB groups could improve the expression of VEGF at different stages of fracture repair, which was consistent to the results of $\mathrm{HE}$ analysis.

In this research, we investigated the effect of $\mathrm{WO}$ on the expression of VEGF during fracture healing in rabbit radius fracture model for the first time. The conclusion conformed to the results of clinical practices. However, there were still some limitations. First, although the positive control of this study, YB is widely utilized to promote fracture healing in China, but it's unacquainted for other researchers. The mechanisms concerning how YB accelerated fracture healing were also not widely reported. Second, there are some other proteins such as bone morphogenetic protein 2, osteocalcin and type I collagen that also contributing to fracture healing which were not studied in the present research. Whether WO or YB affects expressions of those factors during the treatment still needs other evidences. Moreover, the real conditions of fracture were different from the experimental condition. The causes for bone fractures are very complex and there are great individual differences among patients. Fractures are associated with different complications such as soft tissue damage, massive bleeding, severe pain, infection, visceral injury and even shock. Thus, experiments in animals are not enough and the mechanism of WO on the treatment of fracture needs more studies.

\section{Conclusions}

In conclusion, we had detected the composition of WO and confirmed that its effect on increasing the VEGF expression in the process of bone regeneration was good for neovascularization and callus regeneration, as well as acceleration of fracture healing. This research will deepen our understanding of the function of WO, and will be of value in the development and application of WO.

\section{Additional files}

Additional file 1. The minimum standards of reporting checklist.

Additional file 2: Figure S1. The structures of the active compounds obtained by LC-MS were shown.

\begin{abstract}
Abbreviations
ALP: alkaline phosphatase; DAB: diaminobenzidine; EDTA: ethylene diamine tetraacetic acid; FBXO32: F-box protein 32; GAPDH: glyceraldehyde-3-phosphate dehydrogenase; HE: hematoxylin-eosin staining; PVDF: polyvinylidene fluoride; RIPA: radio-immunoprecipitation assay; SDS-PAGE: sodium dodecyl sulfate-polyacrylamide gel electrophoresis; VEGF: vascular endothelial growth factor; WO: wound ointment; YB: Yunnan Baiyao; YNBY: Yunnan Baiyao; LC-MS: liquid chromatograph-mass spectrometer; ELISA: enzyme linked immunosorbent assay.
\end{abstract}

\section{Authors' contributions}

Study design and conception: ZO, QC; data collection: ZO, QC, YC, TC; data analysis: ZO, QC, XR, FL, XZ, QL; data interpretation: ZO, QC, FL, XZ, QL; manuscript drafting: ZO, QC; critical revising the manuscript: ZF. All authors read and approved the final manuscript.

\section{Author details}

${ }^{1}$ Department of Traumatic Orthopedics and Hand Surgery, Ruikang Hospital Affiliated to Guangxi University of Chinese Medicine, Block B, No 10 Huadong Road, Nanning 530011, Guangxi, China. ${ }^{2}$ Department of Traumatic Orthopedics, Daye Hospital of Traditional Chinese Medicine, Daye 435100, Hubei, China.

\section{Acknowledgements \\ Not applicable. \\ The work was done in Department of Traumatic Orthopedics and Hand Surgery, Ruikang Hospital Affiliated to Guangxi University of Chinese Medicine, Nanning, 530011, Guangxi, China.}

\section{Competing interests}

The authors declare that they have no competing interests.

Availability of data and materials

Please contact author for data requests.

Consent for publication

Not applicable.

Ethics approval and consent to participate

This study was approved by the Ethical Committee of Ruikang Hospital Affiliated to Guangxi University of Chinese Medicine.

\section{Funding}

This study was supported by Guangxi Zhuang Autonomous Region Science and Technology Agency Research Project (No. 0816004-37).

\section{Publisher's Note}

Springer Nature remains neutral with regard to jurisdictional claims in published maps and institutional affiliations.

Received: 1 August 2017 Accepted: 9 October 2017

Published online: 30 October 2017

\section{References}

1. Dombrowski S, Jacob L, Hadji P, Kostev K. Oral contraceptive use and fracture risk-a retrospective study of 12,970 women in the UK. Osteoporos Int. 2017;28(8):2349-55. 
2. Cunningham TD, Martin BC, DeShields SC, Romero CC. The impact of osteoporotic fractures compared with other health conditions in older adults living in Virginia, United States. Osteoporos Int. 2016;27:2979-88.

3. Benetou V, Orfanos P, Feskanich D, Michaelsson K, Pettersson-Kymmer $U$, Eriksson S, et al. Fruit and vegetable intake and hip fracture incidence in older men and women: the CHANCES project. J Bone Miner Res. 2016:31:1743-52.

4. Ogilvie CM, Lu C, Marcucio R, Lee M, Thompson Z, Hu D, et al. Vascular endothelial growth factor improves bone repair in a murine nonunion model. lowa Orthop J. 2012;32:90-4.

5. Karres J, Heesakkers NA, Ultee JM, Vrouenraets BC. Predicting 30-day mortality following hip fracture surgery: evaluation of six risk prediction models. Injury. 2015;46:371-7.

6. Du B, Liu W, Deng Y, Li S, Liu X, Gao Y, et al. Angiogenesis and bone regeneration of porous nano-hydroxyapatite/coralline blocks coated with rhVEGF165 in critical-size alveolar bone defects in vivo. Int J Nanomed. 2015;10:2555-65.

7. Grassmann JP, Schneppendahl J, Sager M, Hakimi AR, Herten M, Loegters $T T$, et al. The effect of bone marrow concentrate and hyperbaric oxygen therapy on bone repair. J Mater Sci Mater Med. 2015;26:5331.

8. Katagiri W, Kawai T, Osugi M, Sugimura-Wakayama Y, Sakaguchi K, Kojima T, et al. Angiogenesis in newly regenerated bone by secretomes of human mesenchymal stem cells. Maxillofac Plast Reconstr Surg. 2017;39:8.

9. Sukul M, Nguyen TB, Min YK, Lee SY, Lee BT. Effect of local sustainable release of BMP2-VEGF from nano-cellulose loaded in sponge biphasic calcium phosphate on bone regeneration. Tissue Eng Part A. 2015;21:1822-36.

10. Frederick J, Boysen S, Wagg C, Chalhoub S. The effects of oral administration of Yunnan Baiyao on blood coagulation in beagle dogs as measured by kaolin-activated thromboelastography and buccal mucosal bleeding times. Can J Vet Res. 2017;81:41-5.

11. Murphy LA, Panek CM, Bianco D, Nakamura RK. Use of Yunnan Baiyao and epsilon aminocaproic acid in dogs with right atrial masses and pericardial effusion. J Vet Emerg Crit Care (San Antonio). 2017;27:121-6.

12. Li R, Alex P, Ye M, Zhang T, Liu L, Li X. An old herbal medicine with a potentially new therapeutic application in inflammatory bowel disease. Int J Clin Exp Med. 2011;4:309-19.

13. Zhou J, Ni M, Liu X, Ren Z, Zheng Z. Curcumol promotes vascular endothelial growth factor (VEGF)-mediated diabetic wound healing in streptozotocin-induced hyperglycemic rats. Med Sci Monit. 2017;23:555-62.

14. Tang QL, Han SS, Feng J, Di JQ, Qin WX, Fu J, et al. Moist exposed burn ointment promotes cutaneous excisional wound healing in rats involving VEGF and bFGF. Mol Med Rep. 2014;9:1277-82.
15. Ho TJ, Jiang SJ, Lin GH, LiTS, Yiin LM, Yang JS, et al. The in vitro and in vivo wound healing properties of the Chinese herbal medicine "Jinchuang Ointment". Evid Based Complement Alternat Med. 2016;2016:1654056.

16. Arti $\mathrm{H}$, Khorami M, Ebrahimi-Nejad V. Comparison of negative pressure wound therapy (NPWT) \& conventional wound dressings in the open fracture wounds. Pak J Med Sci. 2016;32:65-9.

17. Yang GE, Duan X, Lin D, Li T, Luo D, Wang L, et al. Rapamycin-induced autophagy activity promotes bone fracture healing in rats. Exp Ther Med. 2015;10:1327-33.

18. Murphy CM, Schindeler A, Cantrill LC, Mikulec K, Peacock L, Little DG. PTH(1-34) treatment increases bisphosphonate turnover in fracture repair in rats. J Bone Miner Res. 2015;30:1022-9.

19. Ke K, Li Q, Yang X, Xie Z, Wang Y, Shi J, et al. Asperosaponin VI promotes bone marrow stromal cell osteogenic differentiation through the PI3K AKT signaling pathway in an osteoporosis model. Sci Rep. 2016;6:35233.

20. Berendsen AD, Pinnow EL, Maeda A, Brown AC, McCartney-Francis N, Kram $V$, et al. Biglycan modulates angiogenesis and bone formation during fracture healing. Matrix Biol. 2014;35:223-31.

21. Ozturk BY, Inci I, Egri S, Ozturk AM, Yetkin H, Goktas G, et al. The treatment of segmental bone defects in rabbit tibiae with vascular endothelial growth factor (VEGF)-loaded gelatin/hydroxyapatite "cryogel" scaffold. Eur J Orthop Surg Traumatol. 2013;23:767-74.

22. Song N, Zhao Z, Ma X, Sun X, Ma J, Li F, et al. Naringin promotes fracture healing through stimulation of angiogenesis by regulating the VEGF/ VEGFR-2 signaling pathway in osteoporotic rats. Chem Biol Interact. 2017;261:11-7.

23. Hu K, Olsen BR. Osteoblast-derived VEGF regulates osteoblast differentiation and bone formation during bone repair. J Clin Invest. 2016;126:509-26.

24. Stegen S, van Gastel N, Carmeliet G. Bringing new life to damaged bone: the importance of angiogenesis in bone repair and regeneration. Bone. 2015;70:19-27.

25. Issa JP, Ingraci de Lucia C, Dos Santos Kotake BG, Goncalves Gonzaga M, Tocchini de Figueiredo FA, Mizusaki lyomasa D, et al. The effect of simvastatin treatment on bone repair of femoral fracture in animal model. Growth Factors. 2015;33:139-48.

26. Lee JY, Son SJ, Son JS, Kang SS, Choi SH. Bone-healing capacity of PCL/ PLGA/Duck beak scaffold in critical bone defects in a rabbit model. Biomed Res Int. 2016;2016:2136215.

27. De Kock T, Boone MA, De Schryver T, Van Stappen J, Derluyn H, Masschaele $B$, et al. A pore-scale study of fracture dynamics in rock using $\mathrm{X}$-ray micro-CT under ambient freeze-thaw cycling. Environ Sci Technol. 2015;49:2867-74

\section{Submit your next manuscript to BioMed Central and we will help you at every step:}

- We accept pre-submission inquiries

- Our selector tool helps you to find the most relevant journal

- We provide round the clock customer support

- Convenient online submission

- Thorough peer review

- Inclusion in PubMed and all major indexing services

- Maximum visibility for your research

Submit your manuscript at www.biomedcentral.com/submit 